SLEEP

\section{Play it again}

Repetition is the mother of learning, states an old Latin proverb. Recent studies in rodents have shown that the sequence in which hippocampal

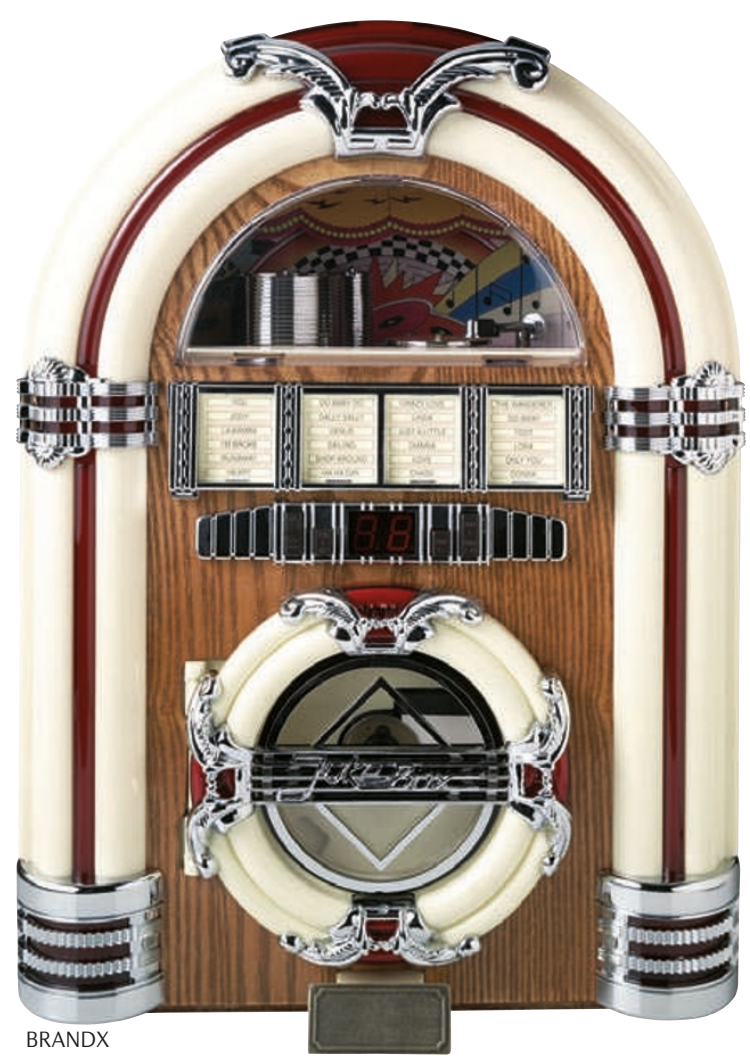

and cortical neurons fire during an experience is replayed

during slow-wave sleep. This replay is assumed to contribute to memory consolidation. Now, Karlsson and Frank demonstrate the existence of replay of previous, spatially remote experiences during waking ("awake remote replay") and Peyrache et al. show that during sleep, the neural firing pattern that appears during new learning is replayed in the medial prefrontal cortex (mPFC) in concert with hippocampal activity.

In the first paper, Karlsson and Frank monitored the firing patterns of hippocampal place cells in two different environments. Between trials animals were placed in a rest box. When in the rest box after trials in environment 1 , the rats frequently showed replay of place cell activation sequences from their experience in environment 1 . Surprisingly, these sequences were more prevalent when the animals were awake than when they were in a sleep-like, quiescent state. This awake replay of environment 1 was also seen when animals paused between bouts of movement in environment 2 . Thus, the hippocampus can replay past, remote experiences during waking. These findings suggest that awake replay might have a particularly important role in memory consolidation.
Peyrache et al. investigated replay by recording in both the hippocampus and the neocortex during a learning experience in a Y maze and in subsequent sleep. In each trial, rats had to select the arm of the maze that contained a reward, but the position of the reward arm changed during the task, so that the animals had to learn a new rule to keep obtaining the reward.

First, the authors recorded patterns of neural activation in the mPFC during rule learning. They then observed that during subsequent slow-wave sleep, preferential reactivation of learning-related activation patterns in the mPFC coincided with hippocampal sharp waves or ripples (which have been associated with hippocampal replay), suggesting that hippocampal-neocortical interactions were taking place.

These studies show that replay of neuronal activation patterns occurs during sleep after learning experiences and also during quiet waking, providing more evidence that neuronal reactivation underlies learning and memory.

Claudia Wiedemann

ORIGINAL RESEARCH PAPER Karlsson, M. P. \& Frank, L. M. Awake replay of remote experiences in the hippocampus. Nature Neurosci. 14 Jun 2009 (doi:10.1038/nn.2344) | Peyrache, A. et al. Replay of rule-learning related neural patterns in the prefrontal cortex during sleep. Nature Neurosci. 31 May 2009 (doi:10.1038/nn.2337) 